\title{
Artemether Treatment of Prepatent Schistosoma japonicum Induces Resistance to Reinfection in Association with Reduced Pathology
}

\author{
Paul B. Bartley,* Amber Glanfield, Yuesheng Li, Danielle I. Stanisic, Mary Duke, Malcolm K. Jones, and \\ Donald P. McManus \\ Molecular Parasitology and Molecular Immunology Laboratories, and the Co-Operative Research Centre for Vaccine Technology, \\ Division of Immunology and Infectious Diseases, Queensland Institute of Medical Research, Herston, Australia; Hunan Institute for \\ Parasitic Diseases, Yueyang, Hunan Province, PR China; School of Veterinary Sciences, The University of Queensland, Australia; \\ Dept of Infection and Immunity, Walter and Eliza Hall Institute of Medical Research, Parkville, Australia
}

\begin{abstract}
Artemether (ART) is a well-described antimalarial with efficacy against juvenile schistosomes, with 7-dayold schistosomula being particularly susceptible. Both ART-affected worms and parasites developing from irradiated cercariae die at similar times after infection. Our aim was to determine if ART treatment of prepatent schistosomiasis japonica may result in the generation of a protective immune response. Female CBA mice were treated with a single dose of ART at defined time points after percutaneous infection with a virulent Chinese mainland strain of Schistosoma japonicum. Half of the mouse cohorts were subjected to homologous parasite strain reinfection after drug treatment to assess protective effects of ART therapy. Two independent trials demonstrated that a statistically significant (58\% and $50 \%$ ) reduction in challenge worm burden occurred after reinfection of those mice treated with ART at two weeks p.i. A reduction in the IL-4 response to soluble worm antigen preparation (SWAP) was also seen in ART-treated mice but with no correlation to reinfection resistance. In the Chinese mainland strain used, ART treatment of prepatent infection at the appropriate time point induced resistance to reinfection. There was also an anti-pathology effect observed in ART-treated mice that remained significant after reinfection.
\end{abstract}

\section{INTRODUCTION}

The activity of artemether (ART), the $\beta$-methyl ether derivative of artemesinin, against Schistosoma japonicum was first described by Cheng and colleagues. ${ }^{1-3}$ Adult worms are largely refractory to the drug, but it is effective against schistosome larvae, ${ }^{4}$ with 7 -day-old schistosomula being particularly susceptible. ${ }^{5}$ ART-affected worms demonstrate vesiculation and peeling of the tegument, adherence of host leukocytes, decreased glycolysis, are smaller (30-50\% reduction in size), and have atrophic testes and ovaries. ${ }^{4,6,7}$ These effects appear to be partly reversible in worms by day 56 postinfection. There is a noticeable shift of affected worms from their usual vascular beds to the liver. ${ }^{4,8}$ Consistent with an anti-fecundity effect, liver granuloma frequency and severity of fibrosis are reduced in ART-treated mice, rabbits, and dogs. ${ }^{9}$ Worm burden reduction rates resulting from 1-day to 4-day treatment courses range from $50 \%$ in mice to $>90 \%$ in rabbits. ${ }^{9}$

There are two major goals in the control of schistosomiasis, a disease still of major public health importance in over 70 countries of the tropics and sub-tropics, and ART may contribute toward achieving both. The first is the development of alternative therapies to praziquantel (PZQ) in view of the potential for induction of tolerance and/or resistance in schistosomes. Encouragingly, a recently completed double-blind field trial on the effects of artemether on S. japonicum infection in a highly endemic focus in southern China confirmed earlier studies that oral artemether significantly reduces the prevalence and intensity of patent $S$. japonicum infections. ${ }^{10}$ The second goal is to induce resistance to schistosome infection. We and others ${ }^{11}$ have hypothesized that an antigen or set of antigens on the larval (schistosomulum) migratory stage is exposed to the immune system after artemether-treatment

* Address correspondence to Paul B. Bartley, Molecular Parasitology Laboratory, Queensland Institute of Medical Research, Herston Road, Herston, 4029, Australia. E-mail: paul.bartley@qml.com.au of prepatent schistosomes. This could result in the induction of a protective immune response, in a similar fashion to the irradiated cercarial vaccine model $^{12}$ where attenuated parasites reach early development, but not full maturity. Indeed, by early administration of ART, Bergquist and others ${ }^{11}$ were able to induce a high level of resistance against $S$. mansoni reinfection in mice. In their hands, that was comparable to vaccination with irradiated cercariae. In this report, we describe experiments undertaken to determine whether single dose artemether treatment of prepatent $S$. japonicum infection was able to induce similar levels of resistance to reinfection.

\section{MATERIALS AND METHODS}

Artemether, parasites, and mice. Artemether (ART) powder was obtained from the Kunming Pharmaceutical Corporation (Kunming, PR China) (a gift from S. Xiao, National Institute of Parasitic Diseases-CDC, Shanghai). ART was suspended at a concentration of $20 \mathrm{mg} / \mathrm{mL}$ in a solution of $0.5 \%$ ethanol, $1 \%$ gum tragacanth and $7 \%$ Tween 80 in water. One dose of ART-400 mg/kg- (or solution only) was given to each mouse by gavage feeding at the indicated time point.

Each group in each trial contained eight female CBA mice (Animal Resource Center, Western Australia) weighing 10-20 g at commencement of the trials. These mice are susceptible to $S$. japonicum infection and produce an intermediate degree of egg-induced hepatic fibrosis. ${ }^{13}$

Oncomelania hupensis hupensis snails, infected with Chinese mainland strain S. japonicum (Anhui province), were kindly provided by Guo Jiagang (National Institute of Parasitic Diseases-CDC, Shanghai). Snails were placed in deionized water and exposed to incandescent light for 3 to $4 \mathrm{~h}$. Cercariae, collected from the water surface by a $10 \mu \mathrm{L}$ bacteriological loop, were used to infect the mice.

Infection, treatment, challenge, perfusion, and egg counts. The experimental design for the two experiments (Trial 1 and Trial 2) is shown in Table 1 . A total of 144 mice were used 
TABLE 1

Experimental layout for Trials 1 and 2 indicating the type of the initial infection at week 0, timing of the Artemether administration, challenge infection with cercariae and perfusion of mice. Each group consisted of 8 female CBA mice.

\begin{tabular}{|c|c|c|c|c|c|c|c|c|c|}
\hline \multirow[b]{2}{*}{ Group } & \multirow[b]{2}{*}{ Subgroup } & \multirow[b]{2}{*}{$\mathrm{n}=$} & \multicolumn{7}{|c|}{ Time (week) } \\
\hline & & & 0 & 1 & 2 & 3 & 5 & 8 & 10 \\
\hline \multirow[t]{2}{*}{ Control 1} & A & 8 & 20 cercariae & & & & 50 cercariae & \multirow{3}{*}{ Perfuse } & Perfuse \\
\hline & B & 8 & 20 cercariae & & & & & & \\
\hline Control 2 & & 8 & & & & & 50 cercariae & & Perfuse \\
\hline \multirow[t]{2}{*}{ Art-1 } & A & 8 & 20 cercariae & ART & & & 50 cercariae & \multirow{3}{*}{ Perfuse } & Perfuse \\
\hline & B & 8 & 20 cercariae & ART & & & & & \\
\hline \multirow[t]{2}{*}{ Art-2 } & A & 8 & 20 cercariae & & ART & & 50 cercariae & & Perfuse \\
\hline & B & 8 & 20 cercariae & & ART & & & \multirow{2}{*}{ Perfuse } & \\
\hline \multirow[t]{2}{*}{ Art-3 } & A & 8 & 20 cercariae & & & ART & 50 cercariae & & Perfuse \\
\hline & B & 8 & 20 cercariae & & & ART & & \multicolumn{2}{|l|}{ Perfuse } \\
\hline
\end{tabular}

Art and $\mathrm{ART}=$ Artemether; cercariae $=$ normal cercariae.

between the two trials; $20( \pm 1) S$. japonicum cercariae were administered percutaneously to the shaved abdomen of each mouse for 50 minutes under intraperitoneal (IP) anesthesia with ketamine and xylazil. ART was administered by gavage tube at a dose of $400 \mathrm{mg} / \mathrm{kg}^{4}$ once at 1,2 , or 3 weeks p.i. Blood was collected by tail vein puncture at baseline (day 0 ), prechallenge (week 5), and prior to perfusion (week 8 or week 10), centrifuged at $10000 \times g$ for $5 \mathrm{~min}$ and the serum stored at $-20^{\circ} \mathrm{C}$. Half of the control or treated mice were given a second percutaneous infection of 50 cercariae under anesthesia at week 5 .

At either week 8 or week 10 , mice were anticoagulated with sodium heparin, killed with $\mathrm{CO}_{2}$, perfused with cold PBS, and the intestine dissected to collect worms. Liver portions were collected from individual mice for egg burden determinations $^{14}$ (expressed as eggs per gram of liver per female worm (EPG/female)) and for preservation in 5\% (v/v) paraformaldehyde in PBS for histological analysis.

Paraffin sections of liver samples were stained with Mayer's hematoxylin and eosin to measure egg-induced pathology. Mean granuloma diameter was measured for individual mice, with results reported as a mean and standard deviation of the group. Additionally, liver pathology was quantified by measurement of the volume density of hepatic granulomas by a point-counting method. ${ }^{15,16}$ Granuloma volume density is defined simply as the proportion of liver volume occupied by egg-induced granulomas. To measure this, 5 random photographs ( $\times 50$ magnification) were taken from each of 3 randomly cut liver sections per mouse. Grids generated by image analysis software (ImageJ-NIH, Bethesda) were laid over each image for point counting. Volume density of hepatic granulomas was estimated from the number of grid points intersecting a granuloma divided by the number of points intersecting all liver tissue in the photograph. Counts for each photograph were averaged over photographs within animals and means and standard deviations were calculated for each group.

Immunological investigations. Mouse spleens in Iscove's modified Dulbecco's Medium (IMDM-Gibco BRL, MD) were crushed and cells transferred to a sterile tube and resuspended to $10 \mathrm{~mL}$ with IMDM. Cells were centrifuged $(3000 \times g$ for $8 \mathrm{~min})$, and resuspended to $5 \mathrm{~mL}$ with Geys red cell lysis buffer and incubated on ice for $2 \mathrm{~min}$. Cells were diluted to $20 \mathrm{~mL}$ with IMDM and washed twice (centrifuged $3000 \times g$ for $8 \mathrm{~min}$ ). Cells were resuspended to $6 \mathrm{~mL}$ in IMDM and layered onto a $3 \mathrm{~mL}$ Nycoprep $^{\mathrm{TM}}$ (Axis-Shield PoC AS,
Oslo, Norway) gradient and centrifuged $(4000 \times g$ for $20 \mathrm{~min})$. The buffy coat layer was aspirated and washed twice with 10 mL IMDM. Cells were diluted to a density of $2 \times 10^{6} / \mathrm{mL}$ in IMDM supplemented with $2 \%$ syngeneic mouse serum.

For measurement of cytokine production, $200 \mu \mathrm{L}$ per well aliquots were plated in quadruplicate in 96-well flat-bottom plates (Costar, Corning, NY) and incubated at $37^{\circ} \mathrm{C}$ in $5 \%$ $\mathrm{CO}_{2}$. Cells were stimulated by the addition of either $25 \mu \mathrm{g} / \mathrm{mL}$ of native SWAP (soluble $S$. japonicum adult worm proteins ${ }^{17}$ ) or supplemented IMDM only.

For cytokine production, mouse spleen lymphocyte culture supernatants (above) were collected at 24, 48 and $72 \mathrm{hrs,}$ centrifuged $(1000 \times g$ for $10 \mathrm{~min})$ and stored at $-70^{\circ} \mathrm{C}$. IFN- $\gamma$ and IL-4 levels were determined by antibody-capture ELISA. Maxisorb ELISA plates (Nunc) were coated with anti-mouse IFN- $\gamma$ or anti-mouse IL-4 capture antibodies (BD Biosciences, San Diego, CA) in $50 \mathrm{mM}$ carbonate buffer. Plates were washed and $200 \mu \mathrm{L}$ of each supernatant (diluted with PBST containing $10 \%$ heat-inactivated fetal calf serum-FCS) was added to the required wells. Plates were incubated at $25^{\circ} \mathrm{C}$ and then washed. Detection antibody with avidin-HRP conjugate diluted in PBST-FCS (BD Biosciences, San Diego, CA) was then added to each well. Plates were then incubated at $25^{\circ} \mathrm{C}$. Plates were washed and OPD added to each well. After $30 \mathrm{~min}$ at $25^{\circ} \mathrm{C}$, OD was measured at $450 \mathrm{~nm}$ using a BioRad ELISA plate reader (Hercules, CA). Each plate included a standard curve based on serial dilutions of recombinant standard IFN- $\gamma$ or IL-4 as required.

Outcome measures. Endpoints for protection against reinfection were expressed as the percentage reductions in worm burdens, liver egg burden per female worm, hepatic granuloma diameter, and hepatic granuloma volume density. ${ }^{16}$ The following formula was used to calculate the percentage worm and egg burden reductions after reinfection:

$$
\text { Percentage protective efficacy }=100 \times\left(1-\left(\frac{A-B}{C}\right)\right)
$$

where $\mathrm{A}=$ mean worm (or egg) burden from the challenged group (e.g., Art -1A), B = mean worm (or egg) burden from the associated unchallenged group (e.g., Art-1B), and $\mathrm{C}=$ mean worm (or egg) burden from the challenge control group (Control-2). For example, using the data from Art-1A and Art-1B from Trial 1 (Table 2) the percentage protection would be: $100 \times(1-(20.6-4.5) / 20.1)=19.9 \approx 20 \%$. 
TABLE 2

Mean $( \pm$ SD) total and female worm burdens in Trials 1 and 2

\begin{tabular}{lccccc}
\hline & \multicolumn{2}{c}{ Trial 1} & & \multicolumn{2}{c}{ Trial 2} \\
\cline { 2 - 3 } \cline { 5 - 6 } \cline { 5 - 6 } & Total Worms & Females & & Total Worms & Females \\
\hline Control-1A & $24.3 \pm 10.0$ & $5.5 \pm 1.9$ & & $23.0 \pm 12.8$ & $9.0 \pm 6.1$ \\
Control-1B & $10.8 \pm 5.6$ & $4.2 \pm 2.1$ & & $7.6 \pm 5.3$ & $4.0 \pm 2.4$ \\
Control-2 & $20.1 \pm 7.0$ & $4.1 \pm 1.8$ & & $28.5 \pm 10.8$ & $11.5 \pm 4.6$ \\
Art-1A & $20.6 \pm 5.3$ & $4.9 \pm 2.2$ & & $30.4 \pm 6.1$ & $14.0 \pm 2.8$ \\
Art-1B & $4.5 \pm 3.3$ & $1.3 \pm 1.2$ & & $0.8 \pm 1.0$ & $0.5 \pm 0.5$ \\
Art-2A & $19.1 \pm 5.8$ & $3.6 \pm 0.8$ & & $19.8 \pm 11.0$ & $9.3 \pm 5.8$ \\
Art-2B & $10.6 \pm 3.0$ & $3.6 \pm 1.5$ & & $5.5 \pm 4.4$ & $2.0 \pm 2.0$ \\
Art-3A & $22.6 \pm 9.3$ & $6.1 \pm 2.5$ & $30.8 \pm 10.0$ & $13.7 \pm 4.2$ \\
Art-3B & $8.3 \pm 5.5$ & $2.8 \pm 2.1$ & & $7.9 \pm 1.8$ & $1.5 \pm 0.93$ \\
GIC-A & $12.4 \pm 10.2$ & $2.9 \pm 2.3$ & & $34.5 \pm 8.3$ & $12.5 \pm 5.3$ \\
GIC-B & 0 & 0 & & 0 & 0 \\
\hline
\end{tabular}

\section{RESULTS}

Parasitological outcomes-worm and hepatic egg burdens. Mean total worm and female worm burdens from both trials are detailed in Table 2. Consistent results were achieved for most groups in both trials with some exceptions. The mean worm burden for Art-1B in Trial 2 was significantly lower than for Trial $1(P<0.05)$. The only significant difference in female worm numbers between trials was for Art-2A having fewer females in Trial 1 than Trial $2(P<0.05)$.

Table 3 displays the mean $( \pm$ SD) egg burdens per female worm (expressed as eggs per gram of liver per female worm-EPG/female). There were no statistically significant differences in egg burdens per female for any group between the two trials.

Adult parasite morphology in ART-treated baseline infections. Morphology measurements were obtained for worms perfused in Trial 2 from groups Control-1B, Control-2, Art1B, Art-2B, and Art-3B (Table 4). Male worms from Art-1B and Art-3B were significantly longer compared with the agematched Control-1B group $(P<0.05$ for each comparison; two-tailed $t$ test). Testis diameter was also significantly greater for these two groups $(P<0.05$ for each comparison; two-tailed $t$ test). There was a statistically significant reduction in female worm length in Art-2B and Art-3B $(P<0.05$ for each comparison; two-tailed $t$ test). A significant increase in male length was seen in Art-1B and Art-3B, and also testis length was significantly increased in Art-2B and Art-3B $(P<$ 0.05 for each comparison; two-tailed $t$ test). Morphological measurements of worms from challenged animals were not

TABLE 3

Mean ( \pm SD) hepatic egg burdens (expressed as eggs per gram of liver per female worm; EPG/female) for Trials 1 and 2

\begin{tabular}{llc}
\hline \multicolumn{1}{c}{ Group } & \multicolumn{1}{c}{ Trial 1} & Trial 2 \\
\hline Control-1A & $2400 \pm 2265$ & $2302 \pm 915$ \\
Control-1B & $5378 \pm 3736$ & $7405 \pm 5739$ \\
Control-2 & $2044 \pm 1461$ & $2800 \pm 1310$ \\
Art-1A & $3936 \pm 2257$ & $3638 \pm 1209$ \\
Art-1B & $5074 \pm 6047$ & $3942 \pm 2707$ \\
Art-2A & $2081 \pm 1712$ & $2866 \pm 1642$ \\
Art-2B & $2862 \pm 2538$ & $1780 \pm 997$ \\
Art-3A & $2395 \pm 1222$ & $2546 \pm 570$ \\
Art-3B & $1706 \pm 969$ & $2646 \pm 2733$ \\
GIC-A & $1084 \pm 1002$ & $2615 \pm 1032$ \\
GIC-B & 0 & 0 \\
\hline
\end{tabular}

obtained, due to the inability to definitively distinguish between worms from the primary and challenge infection.

Female worms in Control-2 increased significantly in length by week 8 post-infection (Control-1B) $(P<0.05$; two-tailed $t$ test); ovarian dimensions did not change but the number of eggs within the uterus increased significantly. ART treatment did not result in a significant change in ovarian dimensions although female worm body length was significantly reduced in Art-2B and Art-3B compared with Control-1B $(P<0.05$ for each comparison; two-tailed $t$ test). A significant reduction in egg numbers (visible within the uterus) was observed in Art-1B and Art-3B but not Art-2B compared with Control-1B.

Worm and liver egg burdens in ART-treated baseline infections. Table 5 displays the percentage worm burden reductions after ART treatment of mice given the baseline infection only. For statistical purposes, comparisons were made with the respective Control-1B group for each trial. Art-1B demonstrated significant worm burden reductions of $58 \%$ in Trial 1 and $90 \%$ in Trial $2(P<0.05-0.02$ for each comparison; two-tailed $t$ test). There was no worm burden reduction after ART treatment in groups Art-2B and Art-3B in either trial.

There were statistically significant egg burden reductions of $68 \%$ and $70 \%$ in Art-3B $(P<0.05$; two-tailed $t$ test $)$ compared with respective controls in each trial. There was no statistically significant egg reduction seen in Art-1B or Art-2B in either trial.

Liver disease burden (displayed as mean granuloma diameter and hepatic granuloma volume density) reductions in Trial 2 after ART treatment are displayed in Figure 1 (Panels $\mathrm{A}$ and $\mathrm{C}$ ). The results are typical of those also obtained in Trial 1 (data not shown). There were highly significant mean granuloma diameter reductions from $0.61 \mathrm{~mm}$ in Control-1B to $0.36 \mathrm{~mm}$ in Art-1B $(P<0.0001$; two-tailed $t$ test $), 0.44 \mathrm{~mm}$ in Art-2B $(P<0.0001$; two-tailed $t$ test $)$, and $0.29 \mathrm{~mm}$ in Art-3B $(P<0.0001$; two-tailed $t$ test $)$. Highly significant reductions in hepatic granuloma volume density were also observed. Mean section volume occupied by granuloma was reduced from $42.3 \%$ in Control-1B to $4.7 \%$ in Art-1B, $4.7 \%$ in Art-2B and $2.6 \%$ for Art-3B $(P<0.0001$ for each comparison with Control-1B-Likelihood ratio).

Results of parasite challenge after ART treatment. After homologous strain reinfection, there is no objective method to discriminate between $S$. japonicum worms arising from either the primary or challenge infection. Therefore, the assumption was made that all worms identified in a particular "B" group (e.g., Control-1B) were present in the associated "A" group (in this example, Control-1A) (Table 1).

In both trials, significantly reduced challenge total worm burdens $(58 \%$, Trial 1; 50\%, Trial 2) were found in Art-2A but not in Art-1A or Art-3A compared with the challenge control group (Control-2) (Table 6). Vaccination with a single dose of irradiated cercariae did not induce any resistance to reinfection (data not shown). Percentage reductions in female worms post-challenge essentially mirrored those of the total worm burdens for both trials with significant female worm reductions evident only in Art-2A (data not shown).

Post-challenge egg burden reductions compared with the challenge control group (Control-2) are displayed in Table 7. In Trial 1 , Art-2A was the only group to demonstrate a significant post-challenge egg reduction. Significant egg reductions resulted from all 3 ART-treated groups in Trial 2 and also in the untreated control group (Control-1A). 
TABLE 4

Worm morphology measurements of selected groups from Trial 2

\begin{tabular}{|c|c|c|c|c|c|}
\hline \multicolumn{6}{|c|}{ Male } \\
\hline & \multicolumn{2}{|c|}{ Body } & \multicolumn{2}{|c|}{ Testis } & \\
\hline & Length (mm) & Width (mm) & Length (mm) & Width (mm) & \\
\hline Control-2 & $6.825 \pm 1.191$ & $0.325 \pm 0.050$ & $0.350 \pm 0.061$ & $0.125 \pm 0.018$ & \\
\hline Control-1B & $6.3125 \pm 0.427$ & $0.344 \pm 0.043$ & $0.344 \pm 0.047$ & $0.1 \pm 0.000$ & \\
\hline Art-1B & $7.725 \pm 0.256^{*}$ & $0.36 \pm 0.060$ & $0.445 \pm 0.033^{*}$ & $0.115 \pm 0.022$ & \\
\hline Art-2B & $7.615 \pm 1.082$ & $0.405 \pm 0.086$ & $0.425 \pm 0.095$ & $0.130 \pm 0.041$ & \\
\hline Art-3B & $7.325 \pm 0.622 *$ & $0.3 \pm 0.018$ & $0.47 \pm 0.060 *$ & $0.11 \pm 0.022$ & \\
\hline \multicolumn{6}{|c|}{ Female } \\
\hline & \multicolumn{2}{|c|}{ Body } & \multicolumn{2}{|c|}{ Ovary } & Eggs \\
\hline & Length (mm) & Width (mm) & Length (mm) & Width (mm) & $n$ \\
\hline Control-2 & $10.000 \pm 0.820$ & $0.258 \pm 0.014$ & $0.467 \pm 0.029$ & $0.117 \pm 0.014$ & $57.0 \pm 2.6$ \\
\hline Control-1B & $13.563 \pm 1.063$ & $0.250 \pm 0.061$ & $0.569 \pm 0.131$ & $0.144 \pm 0.038$ & $93.0 \pm 18.2$ \\
\hline Art-1B & $11.375 \pm 1.759$ & $0.244 \pm 0.038$ & $0.469 \pm 0.088$ & $0.131 \pm 0.031$ & $46.8 \pm 27.4^{*}$ \\
\hline Art-2B & $11.458 \pm 0.753^{*}$ & $0.325 \pm 0.043$ & $0.492 \pm 0.052$ & $0.133 \pm 0.014$ & $67.3 \pm 3.8$ \\
\hline Art-3B & $9.858 \pm 0.376^{*}$ & $0.217 \pm 0.052$ & $0.45 \pm 0.090$ & $0.125 \pm 0.025$ & $51.3 \pm 13.1 *$ \\
\hline
\end{tabular}

$*=P<0.05$ compared with Control-1B (two-tailed $t$ test).

Post-challenge reduction in mean granuloma diameter and hepatic granuloma volume density in Trial 2 are displayed in Figure 1 (Panels B and D). Similar results were obtained in Trial 1 (data not shown). The mean granuloma diameter for Control-1A was $0.52 \mathrm{~mm}$. All groups were reinfected after either ART-treated baseline infection (Art-1A $=0.29 \mathrm{~mm}$, $P<0.0001$; Art- $2 \mathrm{~A}=0.31 \mathrm{~mm}, P<0.0001$; Art- $3 \mathrm{~A}=0.27 \mathrm{~mm}$, $P<0.0001)$ or vaccination with irradiated cercariae (GIC-A $=$ $0.19 \mathrm{~mm}, P<0.0001)$. These mean granuloma diameters were not significantly different to the mean granuloma diameter of Control-2. Highly significant reductions in hepatic granuloma volume density for mice reinfected after ART treatment were also demonstrated (Figure 1). In Control-1A, 28.5\% of section volume was occupied by granuloma. Statistically significant reductions were demonstrated in Art-1A $(9.2 \%, P<$ $0.01)$, Art-2A (12.7\%, $P<0.01)$, and Art-3A $(9.8 \%, P<0.01)$. There were no statistically significant differences between Control-2 and groups Art-1A, Art-2A, or Art-3A.

Artemether-induced resistance to reinfection is not attributable to drug modification of the host. A control experiment was performed to exclude the possibility that ART-induced reinfection resistance was attributable to drug-modification of the host. ART was administered to a group of eight 10-11 week-old naive female CBA mice 3 weeks prior to infection with $50 \mathrm{~S}$. japonicum cercariae. This replicates weeks 2-10 of group Art-2A (Table 1). A placebo control group of eight mice was infected simultaneously. There were no significant differences in the mean worm burdens, mean hepatic egg burdens, or mean hepatic granuloma volume densities between the mice pre-treated with ART and the control mice (data not shown). On the basis of this experiment, there ap-

TABLE 5

Worm burden reduction (\%) after ART treatment of baseline infection

\begin{tabular}{cccc}
\hline & \multicolumn{3}{c}{ Artemether treatment } \\
\cline { 2 - 4 } Trial & Art-1B & Art-2B & Art-3B \\
\hline Trial 1 & $58^{*}$ & 2 & 24 \\
Trial 2 & $90 \dagger$ & 27 & -4 \\
\hline Compared with respective Control-1B; $* P<0.05 ; \dagger P<0.02$ (Two-tailed $t$ test).
\end{tabular}

peared no reason to implicate host modification in the etiology of ART-induced resistance to reinfection.

Cellular and humoral responses after ART-treatment. Lymphocyte proliferation assays were performed on mouse spleen lymphocytes from all mice in both trials. Although all ART-treated groups had significantly increased proliferative responses to con A than infected controls, no clear correlation with resistance to reinfection could be identified (data not shown). Additionally, no correlation was identified between protection and SWAP-induced lymphocyte proliferative responses.

Humoral immune responses demonstrated expected increases in IgG and IgM in response to SWAP as the infections progressed. No correlation was observed between SWAPspecific IgG1, IgG2a, IgM, or IgE responses and resistance to reinfection in the challenged groups by ELISA or Western blot (data not shown).

SWAP-specific IL-4 production by cultured splenic lymphocytes was quantified by ELISA. Production of IL-4 peaked at $72 \mathrm{~h}$ (data not shown) and the results are, therefore, displayed as the mean cytokine concentration $( \pm$ SD) at $72 \mathrm{~h}$ from both independent trials (Figure 2). IL-4 was produced in trace amounts by lymphocytes incubated in medium only. SWAP-induced IL-4 production was low in all baseline groups, ranging from $37 \mathrm{pg} / \mathrm{mL}$ (Control-1B) to $140 \mathrm{pg} / \mathrm{mL}$ (Art-2B) with no statistically significant comparisons. However, reinfection after ART treatment was associated with a significant fall in SWAP-induced IL-4 from $1138 \mathrm{pg} / \mathrm{mL}$ (Control-1A) and $705 \mathrm{pg} / \mathrm{mL}$ (Control-2) to $22 \mathrm{pg} / \mathrm{mL}$ (Art-1A), $11 \mathrm{pg} / \mathrm{mL}$ (Art-2A), $12 \mathrm{pg} / \mathrm{mL}$ (Art-3A), and $32 \mathrm{pg} / \mathrm{mL}$ (GICA). All comparisons were statistically significant $(P<0.01-$ two-tailed $t$ test) compared with Control-1A and also Control-2. No statistically significant difference in IFN- $\gamma$ production was detectable in response to incubation with either con A or SWAP (data not shown).

\section{DISCUSSION}

This study of ART-treatment of a virulent Chinese mainland strain of $S$. japonicum demonstrated that a single dose of 

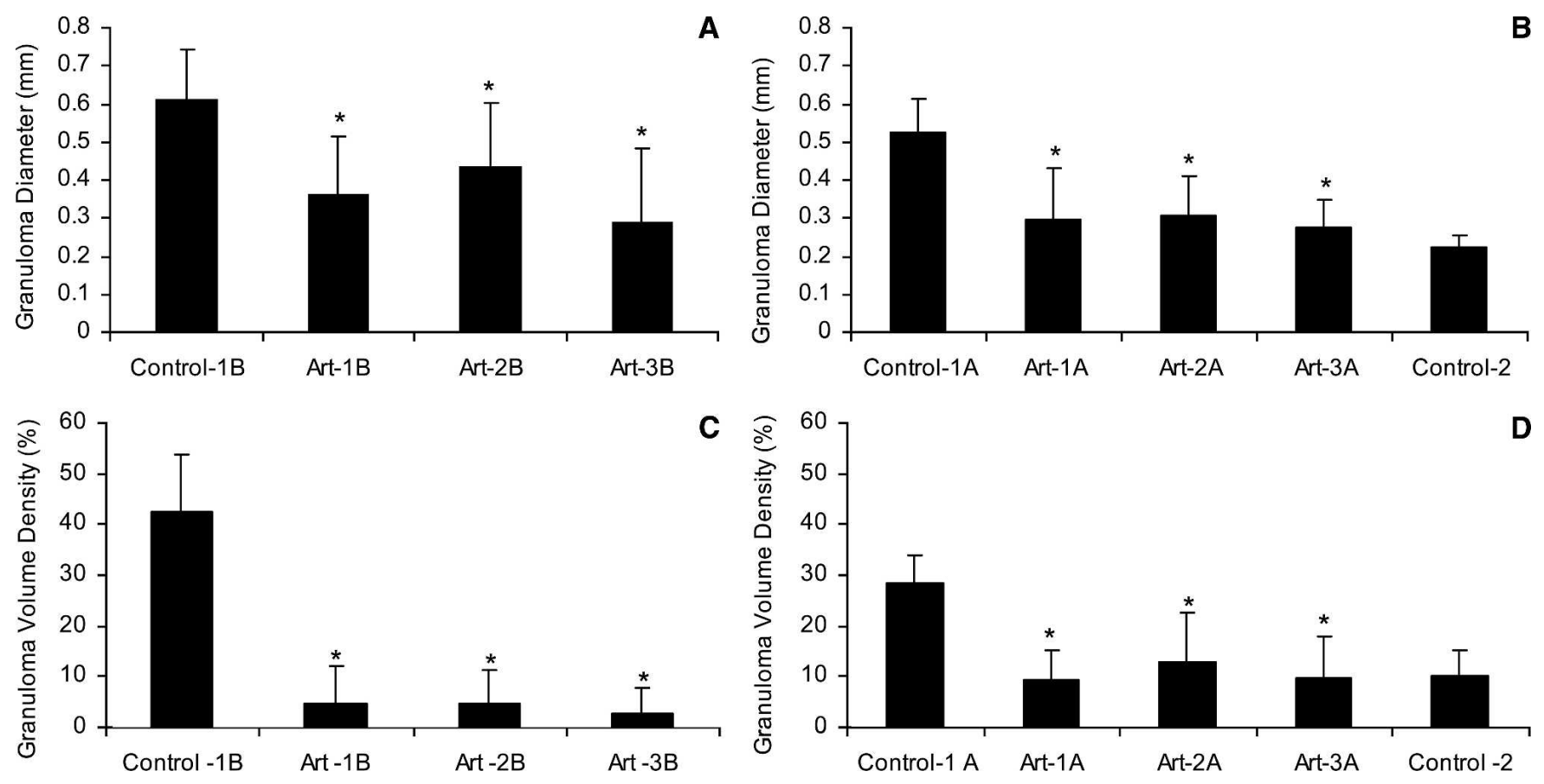

FIGURE 1. Quantification of egg-induced liver pathology by measurement of mean granuloma diameter (in millimeters \pm SD) and hepatic granuloma volume density (expressed as percent of section volume \pm SD). Panels $\mathbf{A}$ and $\mathbf{C}$ display granuloma diameter and hepatic granuloma volume density from the baseline infection groups. Panels $\mathbf{B}$ and $\mathbf{D}$ display data from the groups reinfected following ART-treatment. The two-tailed $t$ test was used for statistical comparisons in panels $\mathbf{A}$ and $\mathbf{B}(*=P<0.0001$ versus Control-1B or Control-1A, respectively). Statistical comparisons in panels $\mathbf{C}$ and $\mathbf{D}$ were made using the Likelihood Ratio Method (* $=P<0.0001$ compared with either Control-1B or Control-1A, respectively). Neither granuloma diameter nor granuloma volume density was significantly different from the challenge control group (Control-2) for mice reinfected after ART treatment.

ART treatment 2 weeks after a baseline infection induced statistically significant resistance $(58$ and $50 \%$ reductions, Trials 1 and 2, respectively) to homologous strain reinfection when worm burden reduction was the primary endpoint. The resistance to reinfection is broadly consistent with those recently reported for $S$. mansoni. ${ }^{11}$ However, we achieved maximum protection at week 2 p.i. for $S$. japonicum, whereas Bergquist and others ${ }^{11}$ demonstrated peak resistance occurring at week 3 for $S$. mansoni. Reinfection of control mice (Control-1A) did not cause a statistically significant reduction in worm burdens in either trial. This is consistent with previous studies ${ }^{18,19}$ where mice infected with S. japonicum were reinfected 5 weeks later without reduction of the challenge worm burden. Maximal reinfection resistance occurred after our measured peak in schistosomulum susceptibility to ART killing when presumably the injured schistosomula are exposed to host immune responses within the lungs.
In our hands, and consistent with previous reports, ${ }^{4,9} 7$-dayold juvenile worms were most susceptible to ART killing (Table 5). We also demonstrated that ART treatment of a single baseline infection caused growth retardation particularly in surviving female worms. Although we were unable to demonstrate ovarian atrophy, the reduced number of eggs produced would be consistent with previous observations demonstrating ovarian toxicity in female worms that survived ART treatment. ${ }^{4,9}$ It has also been postulated that the surviving worms may be able to reverse ART-induced injury. ${ }^{4,9}$ Our study was not designed to specifically evaluate recovery. However, ART treatment at week 3 followed by perfusion at week 8 only allowed 5 weeks for surviving worms to recover, whereas treatment at week 1 allowed 2 additional weeks for recovery. Reduced recovery time may explain the maturation differences observed after ART-treatment at the various time points.

TABLE 6

Protective efficacy (worm burdens) to reinfection with $S$. japonicum in relation to the timing of artemether administration. Protection is expressed as mean percentage worm burden reduction compared with the relevant challenge control group. All comparisons were made using analysis of variance (ANOVA)

\begin{tabular}{|c|c|c|c|c|c|c|}
\hline Trial & Subgroup & ART intervention (p.i.) & Protection (\%) & $95 \%$ C.L. & Z-score & $P$ value \\
\hline \multirow[t]{4}{*}{ Trial 1} & Control-1A & Nil & 31.9 & $(-) 10.7,77.3$ & 1.375 & 1.691 \\
\hline & Art-1A & 1 week & 20.0 & $(-) 10.3,50.6$ & 1.170 & 0.242 \\
\hline & Art-2A & 2 weeks & 57.7 & $31.8,83.6$ & 3.336 & 0.0009 \\
\hline & Art-3A & 3 weeks & 28.9 & $(-) 13.9,71.7$ & 1.227 & 0.2198 \\
\hline \multirow[t]{4}{*}{ Trial 2} & Control-1A & Nil & 44.1 & $(-) 2.5,94.3$ & 1.694 & 0.1 \\
\hline & Art-1A & 1 week & $(-) 3.4$ & $(-) 34.5,27.7$ & -0.256 & 0.798 \\
\hline & Art-2A & 2 weeks & 50.0 & $18.2,81.8$ & 2.489 & 0.0128 \\
\hline & Art-3A & 3 weeks & 19.6 & $(-) 13.9,53.1$ & 1.150 & 0.2501 \\
\hline
\end{tabular}

$95 \% \mathrm{CL}=95 \%$ confidence limits. 
TABLE 7

Protective efficacy (egg burdens) to reinfection with $S$. japonicum in relation to the timing of artemether administration or induced by irradiated cercariae. Protection is expressed as mean percentage egg burden reduction compared with the relevant challenge control group. All comparisons were made using analysis of variance (ANOVA)

\begin{tabular}{llrrrl}
\hline Trial & Intervention & $\%$ Reduction & \multicolumn{1}{c}{$95 \%$ C.L. } & Z-score & $P$ value \\
\hline Trial 1 & Control-1A & 54 & $(-) 187,295$ & 0.743 & 0.458 \\
& Art-1A & 171 & $(-) 53,394$ & 1.479 & 0.1391 \\
& Art-2A & 163 & 53,274 & 2.734 & 0.0063 \\
Trial 2 & Art-3A & 61 & 2,119 & 1.603 & 0.1089 \\
& Control-1A & 158 & $(-) 0.5,321$ & 2.485 & 0.013 \\
& Art-1A & 111 & 38,184 & 2.708 & 0.0068 \\
& Art-2A & 61 & 12,110 & 2.084 & 0.0372 \\
& Art-3A & 104 & 35,173 & 2.653 & 0.008 \\
\hline
\end{tabular}

$95 \% \mathrm{CL}=95 \%$ confidence limits.

The premise of this work is that ART-induced killing or injury of juvenile schistosomes induces resistance to reinfection that is independent of any concomitant immunity or host modification. In our hands, ART-induced reinfection resistance could not be attributed to drug-modification of the host. This was demonstrated by pre-treatment of naïve mice 3 weeks before infection-effectively replicating weeks 2 to 10 of the ART-2A group. Pre-treatment did not influence worm burden, fecundity, or disease volume compared with the appropriate control group.

Control-1A and $-1 \mathrm{~B}$ also account for the possibility of confounding by concomitant immunity. This phenomena is largely attributable to disease-induced portal-systemic shunts causing aberrant maturation and death of immature worms. ${ }^{20-22}$ Disease burden reduction (by $\mathrm{PZQ}$ ) has been demonstrated to negate concomitant immunity. ${ }^{23}$ We decided on a 5-week interval between infections to minimize any confounding effect of portal hypertension in the Control-1A groups. Reducing animal morbidity was also an important ethical consideration in this decision. The reproducible absence of reinfection resistance in Control-1A argues against any significant concomitant immunity in untreated mice. Additionally, we demonstrated that ART treatment of our baseline infection groups effectively reduced disease burden (as determined by both granuloma diameter and hepatic granuloma volume density). Therefore, based on the previous PZQ studies, ${ }^{23}$ this reduced disease burden would have reduced any concomitant immunity effect that could have been present in the reinfected ART-treated groups. For these reasons, we therefore believe that concomitant immunity does not play a significant role in this model.

Therefore, the key difference between Control-2 and ART$1 \mathrm{~A},-2 \mathrm{~A}$, and $-3 \mathrm{~A}$ is the presence of adult worms that survived ART treatment in the latter three groups. The presence of the parallel "B" group in the protection calculation effectively controls for ART killing of worms that occurred in the "A" groups before reinfection. Direct comparisons of total worm burdens between Control-1A and ART-1A, -2A, or -3A are therefore not valid because the effect of ART killing of worms from the baseline infection is not taken into account.

There was a significant reduction in egg production especially after treatment at 3 weeks p.i., with this trend remaining after reinfection. In addition to the effects on egg production, ART treatment caused a significant reduction in both hepatic granuloma diameter and hepatic granuloma volume density.
This anti-pathology effect, combined with the female worm growth retardation discussed above, would support the hypothesis that ART-induced ovarian toxicity caused not only a reduction in egg production, but also a qualitative egg defect.

Interpretation of egg- and disease burdens after reinfection is more complex. Following homologous strain reinfection, Control-1A (compared with Control-2) from Trial 2 demonstrated a significant reduction in liver egg burden in the absence of ART-implying a potential crowding effect. However, an anti-fecundity effect was seen post-challenge for all ART-treatment groups of similar magnitude in Trial 2, and only in Art-3A in Trial 1. Even if, in the context of homologous strain reinfection, ART does not confer a reduction in challenge egg burden, a prominent anti-disease effect persists beyond physical clearance of the drug and appears to have an effect on the subsequent infection. However, unlike the protection from challenge worm burden described above, no one treatment time point appeared to offer specific or maximum protection from disease. This appears to contrast with previous postulations ${ }^{1,2}$ that a fecundity reduction may facilitate improved host immune responses against adult schistosome worms by removing or reducing granuloma-associated $\mathrm{Th}_{2}$ cytokine responses. Peak fecundity and disease reductions in the baseline groups of Trials 1 and 2 occurred consistently after ART-treatment at week 3 p.i. Significant resistance did not occur following reinfection after treatment at this time.

The cytokine response described may contribute toward an explanation of a potential immune mechanism. ART treatment followed by rechallenge appeared to substantially diminish SWAP-induced IL-4 production at all time points. ART treatment did not appear to affect IFN- $\gamma$ responses either to SWAP or concanavalin A before or after reinfection (data not shown). It is possible that ART treatment at week 2 p.i. may interfere with the worm's ability to induce a $\mathrm{Th}_{2}$ immune response, therefore contributing to this reinfection resistance. Although it is difficult to reconcile this data with a known immune mechanism, it appears that impaired SWAPinduced $\mathrm{Th}_{2}$ responses was associated with resistance to reinfection by the Chinese strain of $S$. japonicum in this study.

The protection arising from appropriately timed ARTattenuation of prepatent schistosomiasis japonica appears to parallel that described for $S$. mansoni ${ }^{11}$; it is simple and reproducible. Protection is associated with a loss of SWAPspecific $\mathrm{Th}_{2}$ cytokine responses. The precise mechanism of the immune-mediated protection remains unclear. Additionally, the anti-pathology effect demonstrated after treatment

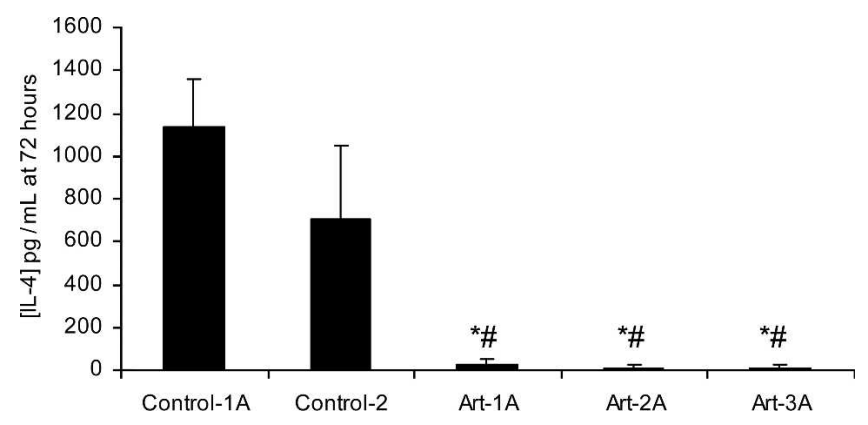

FIGURE 2. IL-4 production by splenic lymphocytes cultured for $72 \mathrm{~h}$ in SWAP. Results are the mean $( \pm 1$ SD) obtained from both Trials 1 and 2 . $*=P<0.01$ Compared with Control-1A; $\#=P<0.01$ compared with Control-2 (two-tailed $t$ test). 
of a prepatent infection persists following homologous strain reinfection. If replicated in human subjects, these two consequences of ART treatment could significantly reduce transmission in field settings. This model also has the additional potential to be a new "gold standard" for the preclinical testing of candidate schistosome vaccines.

Received October 7, 2007. Accepted for publication February 17, 2008.

Acknowledgments: Paul Bartley was supported by a Sir Gustav Nossal Medical Postgraduate Scholarship from the National Health and Medical Research Council (NHMRC) of Australia and a laboratory maintenance grant from the Cooperative Research Centre for Vaccine Technology (CRC-VT), Australia. Financial support from the NHMRC of Australia (DPM and MKJ), Howard Hughes Medical Institute (YL) and the Wellcome Trust (ICRGS Award-DPM) is gratefully acknowledged.

Authors' addresses: Paul B. Bartley, Amber Glanfield, Yuesheng Li, Mary Duke, Malcolm Jones, and Donald McManus, Molecular Parasitology Laboratory, Queensland Institute of Medical Research, Herston Road, Herston, 4029, Australia, Tel: $+61+7+33620401$, Fax: +61 +7 +3362 0104, E-mail: paul.bartley@qml.com.au. Danielle Stanisic, Department of Infection and Immunity, Walter and Eliza Hall Institute of Medical Research, Parkville, Australia.

\section{REFERENCES}

1. Xiao S, Booth M, Tanner M, 2000. The prophylactic effects of artemether against Schistosoma japonicum infections. Parasitol Today 16: 122-126.

2. Xiao S, 2005. Development of antischistosomal drugs in China, with particular consideration to praziquantel and the artemisinins. Acta Trop 96: 153-167.

3. Cheng D, Fu L, Shao P, Wu F, Fang Z, Su H, Ren Z, Sen S, 1980. Studies on chemotherapeutic effect of qinghaosu in experimental schistosomiasis. Chin Med J (Engl) 60: 422-425.

4. Xiao S, Catto B, 1989. In vitro and in vivo studies of the effect of artemether on Schistosoma mansoni. Antimicrob Agents Chemother 33: 1557-1562.

5. Xiao S, Jiqing Y, Jinying M, Huifang G, Peiying J, Tanner M, 2000. Effect of praziquantel together with artemether on Schistosoma japonicum parasites of different ages in rabbits. Parasitol Int 49: 25-30.

6. Xiao S, Shen B, Chollet J, Utzinger J, Tanner M, 2001. Tegumental alterations in juvenile Schistosoma haematobium harboured in hamsters following artemether treatment. Parasitol Int 50: $175-183$.

7. Utzinger J, Xiao S, N'goran E, Bergquist R, Tanner M, 2001. The potential of artemether for the control of schistosomiasis. Int $J$ Parasitol 31: 1549-1562.

8. Sano M, Akyol C, Tungtrongchitr A, Ito M, Ishih A, 1993. Studies on chemotherapy of parasitic helminths: efficacy of artemether on Japanese strain of Schistosoma japonicum in mice. Southeast Asian J Trop Med Public Health 24: 53-56.
9. Xiao S, You J, Yang Y, Wang C, 1995. Experimental studies on early treatment of schistosomal infection with artemether. Southeast Asian J Trop Med Public Health 26: 306-318.

10. Li Y, Chen H, He H, Hou X, Ellis M, McManus D, 2005. A double-blind field trial on the effects of artemether on Schistosoma japonicum infection in a highly endemic focus in southern China. Acta Trop 96: 184-190.

11. Bergquist R, Utzinger J, Chollet J, Shu-Hua X, Weiss N, Tanner $\mathrm{M}$, 2004. Triggering of high-level resistance against Schistosoma mansoni reinfection by artemether in the mouse model. Am J Trop Med Hyg 71: 774-777.

12. Dean D, 1983. Schistosoma and related genera: acquired resistance in mice. Exp Parasitol 55: 1-104.

13. Cheever A, Duvall R, Hallack T Jr, 1984. Differences in hepatic fibrosis and granuloma size in several strains of mice infected with Schistosoma japonicum. Am J Trop Med Hyg 33: 602-607.

14. Cheever A, 1968. Conditions affecting the accuracy of potassium hydroxide digestion techniques for counting Schistosoma mansoni eggs in tissues. Bull WHO 39: 328-331.

15. Bartley PB, Ramm GA, Jones MK, Ruddell RG, Li Y, McManus DP, 2006. A contributory role for activated hepatic stellate cells in the dynamics of Schistosoma japonicum egg-induced fibrosis. Int J Parasitol 36: 993-1001.

16. Griffiths G, 1993. Fine structure immunocytochemistry. Berlin Heidelberg: Springer-Verlag.

17. McManus D, Ross A, Sleigh A, Williams G, Yang W, Li Y, Acosta L, Waine G, 1999. Production of interleukin-10 by peripheral blood mononuclear cells from residents of a marshland area in China endemic for Schistosoma japonicum. Parasitol Int 48: 169-177.

18. Moloney N, Webbe G, 1984. Factors affecting the acquisition of resistance to Schistosoma japonicum in the mouse II. Evidence that resistance to reinfection is not mediated by specific effector mechanisms. Parasitology 89: 361-367.

19. Moloney N, Webbe G, Luty A, 1984. Factors affecting the acquisition of resistance to Schistosoma japonicum in the mouse I. The correlation between egg deposition and worm elimination. Parasitology 89: 345-360.

20. Colley D, Freeman G, 1980. Differences in adult Schistosoma mansoni worm burden requirements for the establishment of resistance to reinfection in inbred mice I. CBA/J and C57BL/6 mice. Am J Trop Med Hyg 29: 1279-1285.

21. Colley D, Freeman G, 1983. Differences in adult Schistosoma mansoni worm burden requirements for the establishment of resistance to reinfection in inbred mice II. C57BL/KsJ, SWR/J, $\mathrm{SJL} / \mathrm{J}, \mathrm{BALB} / \mathrm{cAnN}, \mathrm{DBA} / 2 \mathrm{~N}, \mathrm{~A} / \mathrm{j}, \mathrm{B} 10 . \mathrm{A}(3 \mathrm{R})$, and B10.A(5R) mice. Am J Trop Med Hyg 32: 543-549.

22. McHugh S, Coulson P, Wilson R, 1987. The relationship between pathology and resistance to reinfection with Schistosoma mansoni in mice: a causal mechanism of resistance in chronic infections. Parasitology 94: 81-91.

23. Doenhoff M, Bickle Q, Bain J, Webbe G, Nelson G, 1980. Factors affecting the acquisition of resistance against Schistosoma mansoni in the mouse. V. Reduction in the degree of resistance to reinfection after chemotherapeutic elimination of recently patent primary infections. $J$ Helminthol 54: 7-16. 